\title{
Numerical electromagnetic simulation for high resolution eddy-current testing method
}

\author{
Takuya SAITO $^{1}$, Hitoshi MIKADA ${ }^{1}$, Tadanori GOTO ${ }^{1}$, Junichi TAKEKAWA ${ }^{1}$ \\ ${ }^{1}$ Dept. of Civil and Earth Res. Eng., Kyoto University
}

\begin{abstract}
The applicability and feasibility of eddy current detection method for the measurement of wall thinning and surface crack of steel structure have been practically confirmed by field and laboratory experiments. Recently, we could roughly understand where and how large the defects are by this method. However, it is difficult to estimate the exact size and shape of them. For more accurate inspections, there has been a growing demand to quantitatively evaluate the defects. Therefore, we have developed a numerical simulator to consider whether we could develop the high accuracy eddy current method. Eddy current method uses the information of excitation and induced magnetic field. In order to calculate the induced magnetic field, we used a 2.5 dimensional finite-difference frequency domain technique (2.5D-FDFD) to solve Maxwell's equations numerically. In this technique, we assumed the two-dimensional structure and the three-dimensional electromagnetic field. We used two-layer structure consisting of seawater and steel plate containing defects. To estimate characteristic of the induced magnetic field, we simulated for a variety of defects and compared what effect appear. As a result, we could confirm the effect of surface defects of steel plate on receiving magnetic field intensity. The induced magnetic field intensity increases near the edge of the defects and decays above the defects. The larger depth and width of the defects are, the more attenuate the magnetic field intensity becomes. Our simulation results indicated that we could obtain the response of magnetic field intensity whose detectable scale of defects is no smaller than $\mathrm{mm}$ order.
\end{abstract}

\section{INTRODUCTION}

The applicability and feasibility of eddy current detection method for the measurement of wall thinning and surface crack of steel structure have been practically confirmed by field and laboratory experiments. Recently, we could roughly understand where and how large the defects are by this method although we couldn't estimate the exact size and shape. For more accurate inspections, there has been a growing demand to quantitatively evaluate the defects. Therefore, we have developed a numerical simulator for the investigation regarding more accurate inspections.

Eddy current method uses the information of excitation and induced magnetic field by defects. In order to calculate the induced magnetic field due to the defects, we used a 2.5 dimensional finite-difference frequency domain technique (2.5D-FDFD) to solve Maxwell's equations numerically. In this technique, we assumed the two-dimensional structure and the three-dimensional electromagnetic field. We used two-layer structure consisting of seawater and steel plate containing defects. To estimate characteristic of the induced magnetic field, we simulated for a variety of models with different depth and width of the defects, and investigate the effect of them on the observed data.

\section{THEORY AND METHOD}

\subsection{Maxwell's equation}

In this study, we excite a magnetic field on the surface of the iron, and calculate the secondary magnetic field. To calculate the magnetic field, we use the following equations derived from Maxwell's equation as:

$$
\begin{aligned}
& \nabla \times \nabla \times H+i \omega \mu \hat{\sigma} H=-\hat{\sigma} J_{m}^{s} \\
& \sigma=\sigma+i \omega \varepsilon
\end{aligned}
$$

where $\mathrm{H}[\mathrm{A} / \mathrm{m}]$ is magnetic field intensity, $\mathrm{i}$ is imaginary unit, $\omega$ is angular frequency, $\mu[\mathrm{H} / \mathrm{m}]$ is permeability, $\sigma[\mathrm{S} / \mathrm{m}]$ is conductivity, $\varepsilon[\mathrm{S} / \mathrm{m}]$ is permittivity, $\mathrm{J}[\mathrm{A} / \mathrm{m}]$ is a magnetic source.

\subsection{5D-FDFD method}

Three dimensional Maxwell equations are discretized by a 4th-order finite-difference scheme. Because of the huge matrix, it is very time consuming and needs large memory to solve it. In 


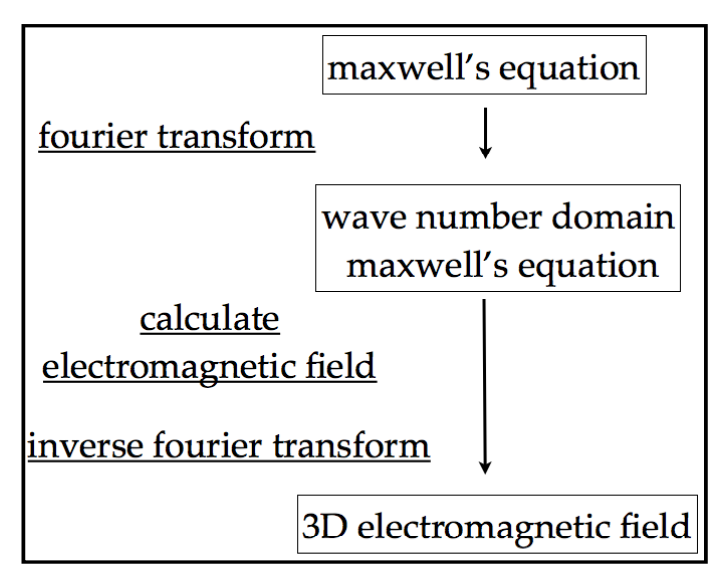

Figure 1: The procedure to calculate 2.5D-FDFD method.

2.5D-FDFD method, we assume two-dimensional structure and three-dimensional electromagnetic field. The procedure to calculate 2.5D-FDFD method is shown in Figure 1.

\subsection{Wave number domain maxwell's equation}

In 2.5D-FDFD method, the electromagnetic field in the $\mathrm{y}$ direction is transformed to wave number domain as below:

$$
\widetilde{H}\left(x, k_{y}, z\right)=\frac{1}{\pi} \int_{0}^{\infty} H\left(x, k_{y}, z, \omega\right) d k_{y}
$$

where $\widetilde{H}$ means wave number domain magnetic field, and $\mathrm{k}_{\mathrm{y}}$ means wave number. Using this equation, the following equations can be derived.

$$
\begin{aligned}
& \left(\mathrm{k}_{\mathrm{y}}^{2}+i \omega \mu \hat{\sigma}-\frac{\partial^{2}}{\partial z^{2}}\right) \widetilde{H}_{x}+i k_{y} \frac{\partial}{\partial x} \widetilde{H}_{y}+\frac{\partial^{2}}{\partial x \partial z} \widetilde{H}_{z}=-\hat{\sigma} J_{x m}^{s} \\
& i k_{y} \frac{\partial}{\partial x} \widetilde{H}_{x}-\left(\frac{\partial^{2}}{\partial x^{2}}+\frac{\partial^{2}}{\partial z^{2}}-i \omega \mu \hat{\sigma}\right) \widetilde{H}_{y}+i k_{y} \frac{\partial}{\partial z} \widetilde{H}_{z}=-\hat{\sigma} J_{y m}^{s} \\
& \frac{\partial^{2}}{\partial x \partial z} \widetilde{H}_{x}+i k_{y} \frac{\partial}{\partial z} \widetilde{H}_{y}+\left(\mathrm{k}_{\mathrm{y}}^{2}+i \omega \mu \hat{\sigma}-\frac{\partial^{2}}{\partial x^{2}}\right) \widetilde{H}_{z}=-\hat{\sigma} J_{z m}^{s}
\end{aligned}
$$

This equation becomes as follows:

$$
\widetilde{A} \widetilde{x}=\widetilde{b}
$$

After solving this equation, the EM fields in the $\mathrm{k}_{\mathrm{y}}$-domain are transformed to the space domain by inverse Fourier transformation. In this study, since the fields at $\mathrm{y}=0$ are taken into consideration, they are evaluated by

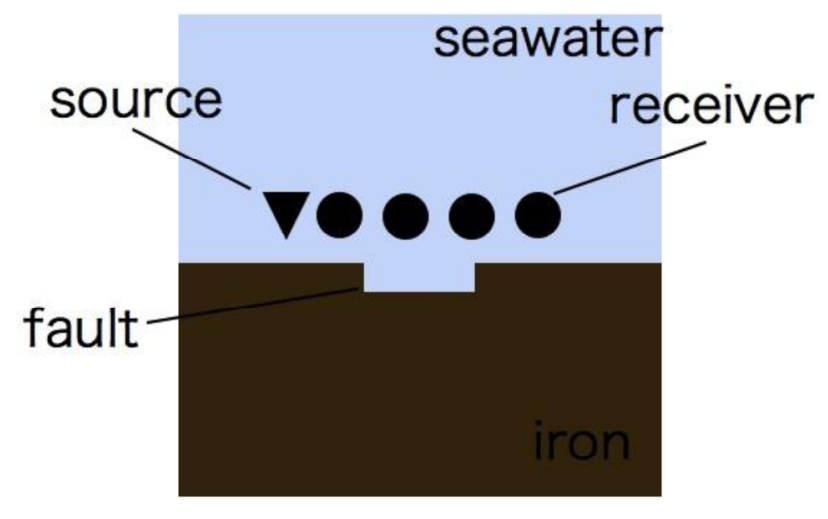

Figure 2: Simulation model A. Triangle and circle points represent source and receivers. The source position is near the edge of crack.

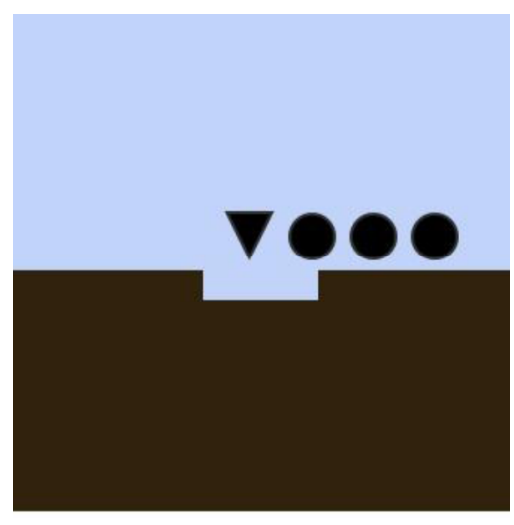

Figure 3: Simulation model B. The source position is above the crack.

$$
H(x, y=0, z, \omega)=\frac{1}{\pi} \int_{0}^{\infty} \widetilde{H}\left(x, k_{y}, z, \omega\right) d k_{y}
$$

This integration is carried out by cubic spline interpolation in the logarithmic wave number domain.

\section{RESULTS AND DISCUSSIONS}

In this section, we examine the effect of the defect on the observed magnetic field. In this study, we use two-layer structure of seawater and conducting material, iron as simulation models (Figure 2, Figure 3).

Figure 4 shows the induced magnetic field intensity calculated with different crack depth from 0 to $7 \mathrm{~mm}$. We can observe that the larger the defects depth are the more attenuate the magnetic field intensity becomes.

Figure 5 shows the induced magnetic field intensity in the case of model A. We observe that the induced magnetic field intensity increases near the edge of the defects and decays above the 


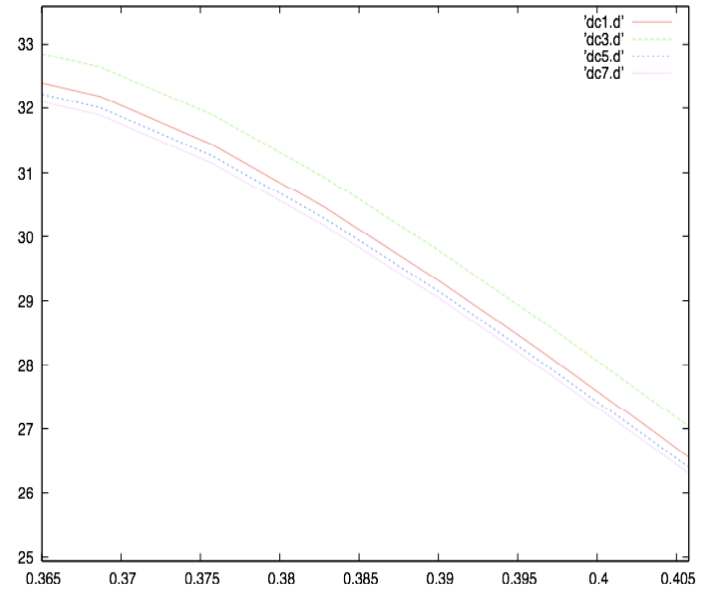

Figure 4: Induced magnetic field intensity calculated while changing crack depth.

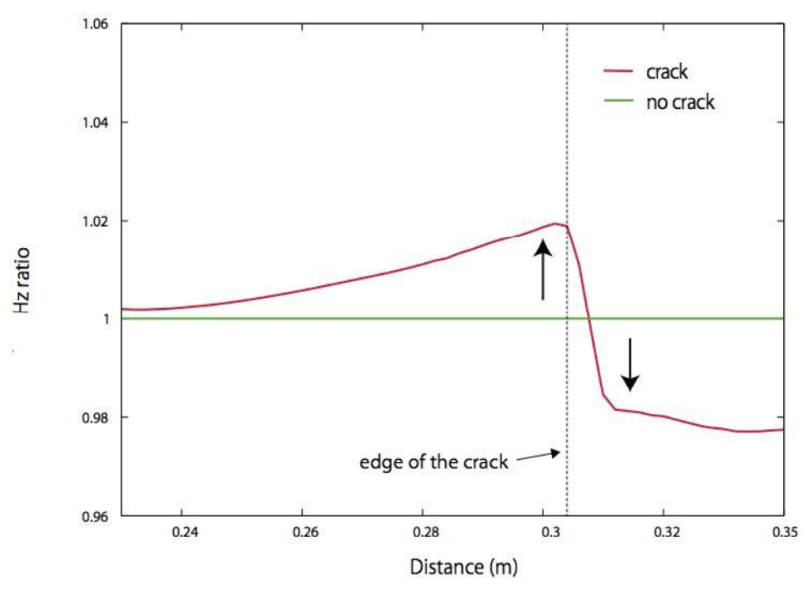

Figure 5: The induced magnetic field intensity in the case of model A. Red line with crack is calculated relative to green line with no crack

defects.

Figure 6 and 7 show the induced magnetic field intensity, in the case of model $\mathrm{B}$, calculated while changing the crack width and depth. We can observe that the larger the defects width and depth are, the more attenuate the magnetic field intensity becomes.

\section{CONCLUSIONS}

We conducted numerical simulations of magnetic field for investigating the applicability and feasibility of eddy current detection method. Our numerical model has a defect which caused the induced magnetic field. The induced magnetic field intensity increases near the edge of the defect and decays above the defect. The larger the defect length and width are, the more attenuate the magnetic field intensity becomes. Our numerical

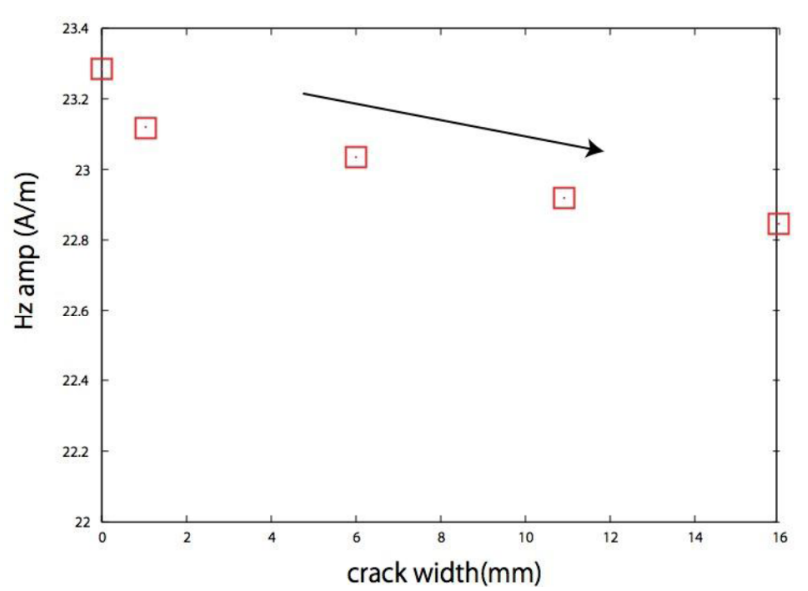

Figure 6: Induced magnetic field intensity in the case of model B calculated while we increase the crack width.

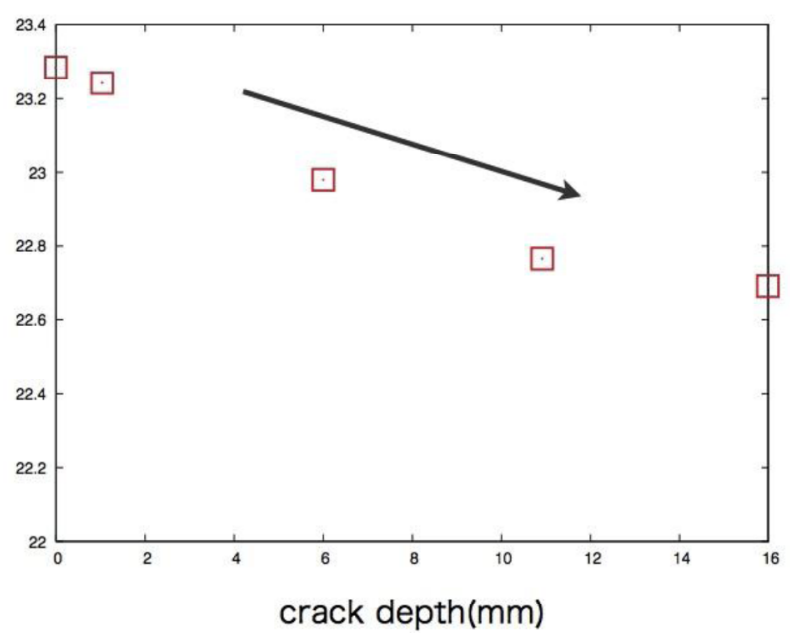

Figure 7: Induced magnetic field intensity in the case of model B calculated while we increase the crack depth.

simulation results indicated that we could calculate the response of magnetic field intensity whose detectable scale of defects is no smaller than $\mathrm{mm}$ order.

\section{REFERENCES}

1) Duck G.Park, Chandra S. ANGANI, Yong M. CHEONG (2012) : Differential Pulsed eddy current probe to detect the sub surface Cracks in a Stainless Steel Pipe, 18th World Conference on Nondestructive Testing

2) Eduardo F. D'Azevedo, Mark R.Fahey, Richard T. Mills (2005) : Vectorized Sparse Matrix Multiply for Compressed Row Storage Format,Lecture Notes in Computer Science, Vol. 3514,99-106

3) E. Haber, U. Ascher (2001) : Fast Finite Volume Simulation of 3D Electromag- netic problems with Highly Discontinuous Coefficients, SIAM Journal on Scientific Computing, Vol. 22, 1943-1961 
4) H. A. Van Der Vorst (1992) : BI-CGSTAB : A first and smoothly converging variant of BI-CG for the solution of nonsymmetric linear systems, SIAM Journal on Scientific Computing, Vol.13, No. 2, 631-644

5) Herrmann,R.B. (1979) : SH-Wave generation by dislocation source, Seismological Society of America, vol. 69, 1-15

6) Leonald J. Bond (2012) : Can new monitoring techniques keep nuclear power station operating safely for 80 years-twice their expected life spans?,IEEE spectrum 8.12, 24-29.

7) Yuji Mitsuhata (2000) : 2-D electromagnetic modeling by finite-element method with a dipole source and topography ,Geophysics, 65, 465-475 